\title{
A Self-organising Model of Market with Single Commodity
}

\author{
Anirban Chakraborti ${ }^{(1)}$, Srutarshi Pradhan ${ }^{(2)}$ and Bikas K. Chakrabarti ${ }^{(3)}$ \\ Saha Institute of Nuclear Physics, \\ 1/AF Bidhan Nagar, Calcutta 700 064, India.
}

\begin{abstract}
We have studied here the self-organising features of the dynamics of a model market, where the agents 'trade' for a single commodity with their money. The model market consists of fixed numbers of economic agents, money supply and commodity. We demonstrate that the model, apart from showing a self-organising behaviour, indicates a crucial role for the money supply in the market and also its self-organising behaviour is seen to be significantly affected when the money supply becomes less than the optimum. We also observed that this optimal money supply level of the market depends on the amount of 'frustration' or scarcity in the commodity market.
\end{abstract}

PACS No. : 87.23.Ge, 05.90.+m, 89.90.+n, 02.50.-r 


\section{Introduction and the model}

An economic market is perhaps the most commonly encountered self-organising system or network, whose dynamics profoundly affects us all. Its dynamics is no doubt very intriguing. In fact Adam Smith in 1776 first made the formal notice of a self-organising aspect, which he called the 'invisible hand' effect, of the market consisting of selfish agents [1, 2]. Although it still remains illusive to demonstrate that a pure competitive market, consisting of agents pursuing pure self-interest, can self-organise or reach (dynamic) equilibrium, the mainstream economists seem to consider it to be true in principle (a matter of faith?) [2]. In various statistical physics models of interacting systems or networks, such self-organisation has indeed been demonstrated to emerge in the global aspects of the system which consists of a large number of simple dynamical elements having local (in time and space) interactions and dynamics [3]. It was also noted by the economists long time back that this dynamics, which takes the system to equilibrium, is greatly facilitated by 'paper money' (rather than the direct commodity exchanges as in barter economy) which does not have any value of its own, but rather can be considered as a good 'lubricant' in the econodynamics [2]. Consistent with this analogy, it was also seen that when the (paper) money supply gets changed, it does not just scale up (for increased money supply) or down (for decreased money supply) the commodity prices, the (self-organising) dynamics towards equilibrium gets seriously affected [4].

We have studied here the self-organising features of the dynamics of a model market, where the agents 'trade' for a single commodity with their money. We demonstrate that the model, apart from having a self-organising behaviour, has got a crucial role for the money supply in the market and that its self-organising behaviour is significantly affected when the money supply becomes less than the optimum. We also observed that this optimal money supply level of the market depends on the amount of 'frustration' or scarcity in the commodity market. In our model, each agent having commodity less than the 'subsistence' level trades with any other having more than the 'subsistence' level, in exchange of its money. Specifically, we consider a closed economic system consisting of $N$ economic agents, each economic agent $i$ have at any time money $m_{i}$ and commodity $q_{i}$, such that $\left(\sum_{i=1}^{N} m_{i}=M\right.$ 
and $\left.\sum_{i=1}^{N} q_{i}=Q\right)$, where $N, M$ and $Q$ are fixed. The 'subsistence' commodity level for each agent is $q_{0}$. Hence at any time an agent having $q_{i}<q_{0}$ will attempt to trade, utilising its money $m_{i}$ at that time, with agents having commodity more than $q_{0}$, and will purchase to make its commodity level $q_{0}$ (and no-further), if its money permits. The agents with $q_{i}>q_{0}$ will sell-off the excess amount to such 'hungry' agents having $q_{i}<q_{0}$, and will attempt to maximize its wealth (money). This dynamics is local in time ('daily') and it stops eventually when no further trade is possible satisfying the above criteria. We introduce an 'annual' or long-time dynamics when some random fluctuations in all the agents' money and commodity occur. Annually, each agent gets a minor reshuffle of the money and its commodity (e.g., perhaps due to the noise in the stock market and in the harvest due to the changes in the weather respectively). This (short and long time) combined dynamics is similar to that of the 'sand-pile' models studied extensively in recent times [3]. The price of the commodity does not change in our model with the money supply $M$ in the market; it remains fixed here (at unity). We look for the steady state features of this market; in particular, the distributions $P(m)$ and $P(q)$ of the money and commodity respectively among the agents. We have investigated how many agents $P\left(q_{0}\right)$ can satisfy their basic needs through this dynamics, i.e., can reach the subsistence level $q_{0}$, as function of the money supply $M$ for both unfrustrated $(g<1)$ (where $g=q_{0} /<q>$ and $<q>=Q / N$ is the average commodity in the market) and the frustrated $(g>1)$ cases of the commodity market. We observed that an optimum amount $M_{0}$ of money supply is required for evolving the market towards the maximum possible value of $P\left(q_{0}\right)$. This optimum value of money $M_{0}$ is observed to decrease with increase in $g$ in the market.

\section{Unlimited money supply and limited supply of commodity :}

Here, we consider the money supply $M$ in the market to be infinitely large and it therefore drops out from any consideration. The dynamics is then entirely governed by the commodity distribution among agents: for agents with $q_{i}<q_{0}$, the attempt will be to find another trade partner having $q_{i}>q_{0}$; and having found such partners, through random search in the market, trades occur for mutual benefit (for the selling agent we still consider the extra money from trade to be important). The system thus evolves towards its steady state, as 
the fixed-point feature of the short-time or daily dynamics gets affected by the random noise reshuffling in the commodity of each agent. This reshuffling essentially induces Gibbs-like distribution $[5,6]$. The trade dynamics is clearly motivated or 'directed'. We look for the combined effect on the steady state distribution of commodity $P(q)$, which is independent of the initial commodity distribution among the agents.

For the unfrustrated case $(g<1)$, where all the agents can be satisfied, the typical distributions $P(q)$ are shown in Fig. 1 for different values of $g$. We see that the $P(q)$ is Gibbs-like $(P(q)=A \exp (-q /<q>)$ and $A=1-g)$, for $q>q_{0}$, while $P\left(q_{0}\right)=g$ (as shown in the inset). One can easily explain these observations using the fact that the cumulative effect of the long-time randomization gives Gibbs distribution $(\exp (-q /<q>)$ for all $q$ ). We then estimate the final steady state distribution $P(q)$ from the additional effect of the short-time dynamics on this (long-time dynamics induced) Gibbs distribution. All the agents with $q<q_{0}$ manage here to acquire $q_{0}$-level of commodity (as $g<1$ and everybody has enough money to purchase the required amount). Their number is then given by $N_{-}=\int_{0}^{q_{0}} \exp (-q) d q$. They require the total amount of commodity $q_{0} N_{-}$. The amount of commodity already available with them is given by $Q_{-}=\int_{0}^{q_{0}} q \exp (-q) d q$. The excess amount required $Q_{\text {demand }}=q_{0} N_{-}-Q_{-}$has to come from the agents having $q>q_{0}$. The average of the excess amount of commodity of the agents who are above the $q_{0}$-line is given by $<q_{\text {excess }}>=\left(1-Q_{-}-\left(1-N_{-}\right) q_{0}\right) /\left(1-N_{-}\right)$. The number of agents who supply the $Q_{\text {demand }}$ amount is given by $N_{+}=Q_{\text {demand }} /<q_{\text {excess }}>$. This gives $P\left(q_{0}\right)=N_{-}+N_{+}=g$. We can easily determine the prefactor of the final steady state distribution $P(q)$ for $q>q_{0}$, $A=1-g$ from the conservation of total number of agents and total commodity.

For the frustrated case $(g>1)$, the results are shown in Fig. 2. A similar calculation for $P\left(q_{0}\right)$ is done as follows: $N_{+}=\int_{q_{0}}^{\infty} \exp (-q) d q$ is the number of people above the $q_{0}$-line who will sell off their excess amount of commodity to come to $q_{0}$-level, $Q_{+}=\int_{q_{0}}^{\infty} q \exp (-q) d q$ is the commodity of the agents above the $q_{0}$-level. Then the supplied amount of commodity to the agents below the $q_{0}$-line is $Q_{\text {supply }}=Q_{+}-q_{0} N_{+}$. The average of the deficit commodity, $<q_{\text {deficit }}>=\left(\left(1-N_{+}\right) q_{0}-1+Q_{+}\right) /\left(1-N_{+}\right)$. Hence, the number of agents who will reach $q_{0}$-level from below is $N_{-}=Q_{\text {supply }} /<q_{\text {deficit }}>$, so that $P\left(q_{0}\right)=N_{+}+N_{-}=g \exp (-g) /(g-$ 
$1+\exp (-g))$. A comparison of this estimate for $P\left(q_{0}\right)$ with $g$ is also shown in the inset of Fig. 2. It may be mentioned that in absence of the strict Gibbs distribution for $P(q)$ ( $\left.q<q_{0}\right)$, the above expression for $P\left(q_{0}\right)$ is somewhat approximate.

\section{Limited money supply and limited supply of commodity :}

When the money supply is limited, the self-organising behaviour is significantly affected and the fraction of agents who can secure $q_{0}$ amount of commodity for themselves $P\left(q_{0}\right)$, does not always reach its maximum possible value (as suggested by the amount of commodity available in the market). For all values of $g$, as we increase the money supply in the market $M, P\left(q_{0}\right)$ increases and then after a certain amount $M_{0}$, it saturates. In Fig. 3., we have shown how the quantity $P\left(q_{0}\right)$ varies with $M$ for different values of $g$ (for $g>1$ only, as we are more interested in the frustrated case). We define $M_{0}$ to be the optimum amount of money supply needed for the smooth functioning of the market. We also observed that this optimal money supply level of the market depends on the amount of frustration $g$ in the commodity market. In the inset, the variation of $M_{0}$ with $g$ is shown for the frustrated case $(g>1)$ only.

\section{Summary and Concluding remarks}

We have studied here the steady state distributions $P(m)$ and $P(q)$ of money and commodity in a model consisting of fixed number of agents $N$, total commodity $Q$ and total money $M$ in the market. Only one commodity is considered for trade and its price is taken to be fixed (at unity) and it does not change with the money supply in the market. The subsistence commodity level $q_{0}$ of all the agents are the same and each of them would like to purchase the deficit amount $\left(q_{0}-q_{i}\right)$ from the agents having $q_{i}>q_{0}$, in exchange of its own money. Apart from the basic urge to reach the subsistence level (if originally below it), all the agents would like to maximize their wealth or money. The second instinct allows the agents with excess commodity (over $q_{0}$ ) to find hungry partners and to sell-off the excess. The dynamics considered here is the local or short time (or daily) dynamics. Additionally, we consider a long time (or yearly) dynamics, which reshuffles mildly but randomly the amounts of money and commodity of each agent (due to, say, the fluctuations in the stock market 
and in the harvest, etc.). The resulting distributions follow from the successive applications of the local directed dynamics, followed by a randomization in the quantities. We consider both the cases: unfrustrated case $(g<1)$ where in principle every agent can be satisfied, and frustrated case $(g>1)$ where not everyone can be satisfied. We concentrate mainly on the quantity $P\left(q_{0}\right)$ which gives the steady density of agents in the market who can satisfy the basic requirement of commodity. As is obvious, this quantity is most significant in the frustrated cases $(g>1)$ where there is not enough commodity in the market to satisfy the basic requirements $\left(q_{0}\right)$ for everyone. We study how this quantity grows or the distribution of commodity among the agents is facilitated, with the supply of money $M$ in the market.

We see that $P\left(q_{0}\right)=g$ for $g<1$ and $P\left(q_{0}\right) \simeq g \exp (-g) /(g-1+\exp (-g))$ for $g>1$, where the money supply $M$ is much greater than $M_{0}$, the optimal money required in the market. We see that these expressions for the $P\left(q_{0}\right)$ for $M>M_{0}$ can be easily explained (see section II) assuming that the resulting distribution is Gibbs like for $q>q_{0}$ (for $g<1$ ) and a mean-field picture (for $g>1$ ). 


\section{References}

e-mail addresses :

(1) anirban@cmp.saha.ernet.in

(2) spradhan@cmp.saha.ernet.in

(3) bikas@cmp.saha.ernet.in

1. A. Smith, An Inquiry into the Nature and Causes of The Wealth of Nations, Strahan and Caddell, London (1776).

2. See e.g., P. A. Samuelson, Economics, 16th Edition, McGraw-Hill Inc., Auckland, 29-32 (1998).

3. See e.g., P. Bak, How Nature Works: The Science of Self-Organised Criticality, Springer, New York (1996).

4. J. M. Keynes, General Theory of Employment, Interest and Money, Royal Economic Society, Macmillan Press, London (1973).

5. A. Dragulescu and V. M. Yakovenko, Eur. Phys. J. B, 17, 723-729 (2000).

6. A. Chakraborti and B. K. Chakrabarti, Eur. Phys. J. B, 17, 167-170 (2000). 


\section{Figure captions}

Fig. 1 : The distributions of commodity $P(q)$ for different values of $g$ for $N=1000, Q=1$, $M=100\left(M>M_{0}(g)\right)$, for the unfrustrated case $(g<1)$. The steady-state distribution of commodity $P(q)$ is Gibbs-like: $P(q)=A \exp (-q /<q>)$ with $A=1-g$, for $q>q_{0}$. The inset shows the linear variation of $P\left(q_{0}\right)$ with $g\left(P\left(q_{0}\right)=g\right)$.

Fig. 2 : The distributions of commodity $P(q)$ for different values of $g$ for $N=1000, Q=1$, $M=100\left(M>M_{0}(g)\right)$, for the frustrated case $(g>1)$. The variation of $P\left(q_{0}\right)$ with $g$ is shown in the inset where the theoretical estimate $(P(q)=g \exp (-g) /(g-1+\exp (-g)))$ is also indicated by the solid line.

Fig. 3 : The variation of quantity $P\left(q_{0}\right)$ with $M$ for different values of $g$ in the frustrated cases $(g>1)$. The inset shows the variation of $M_{0}$ with $g$. 


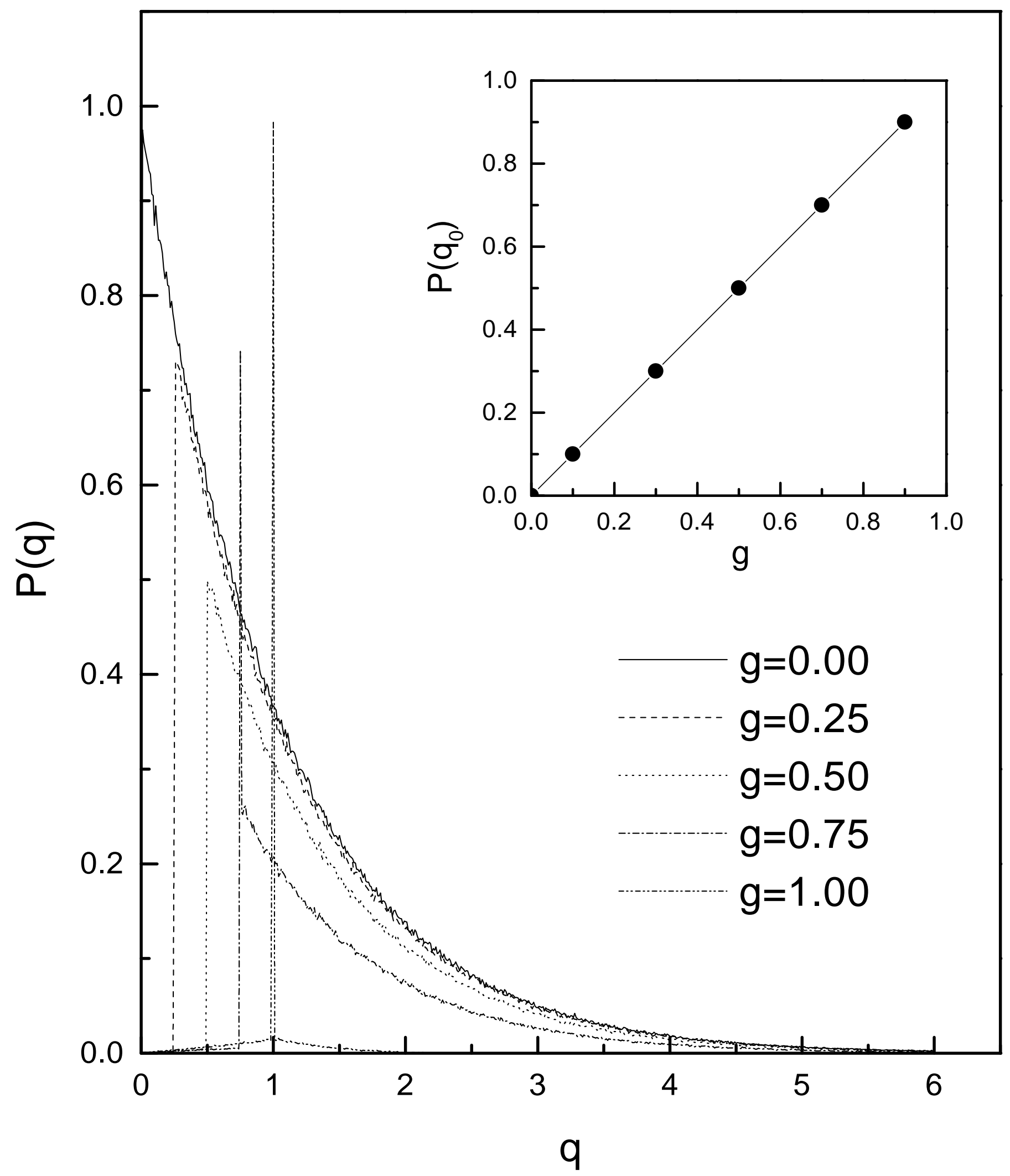

Fig. 1 


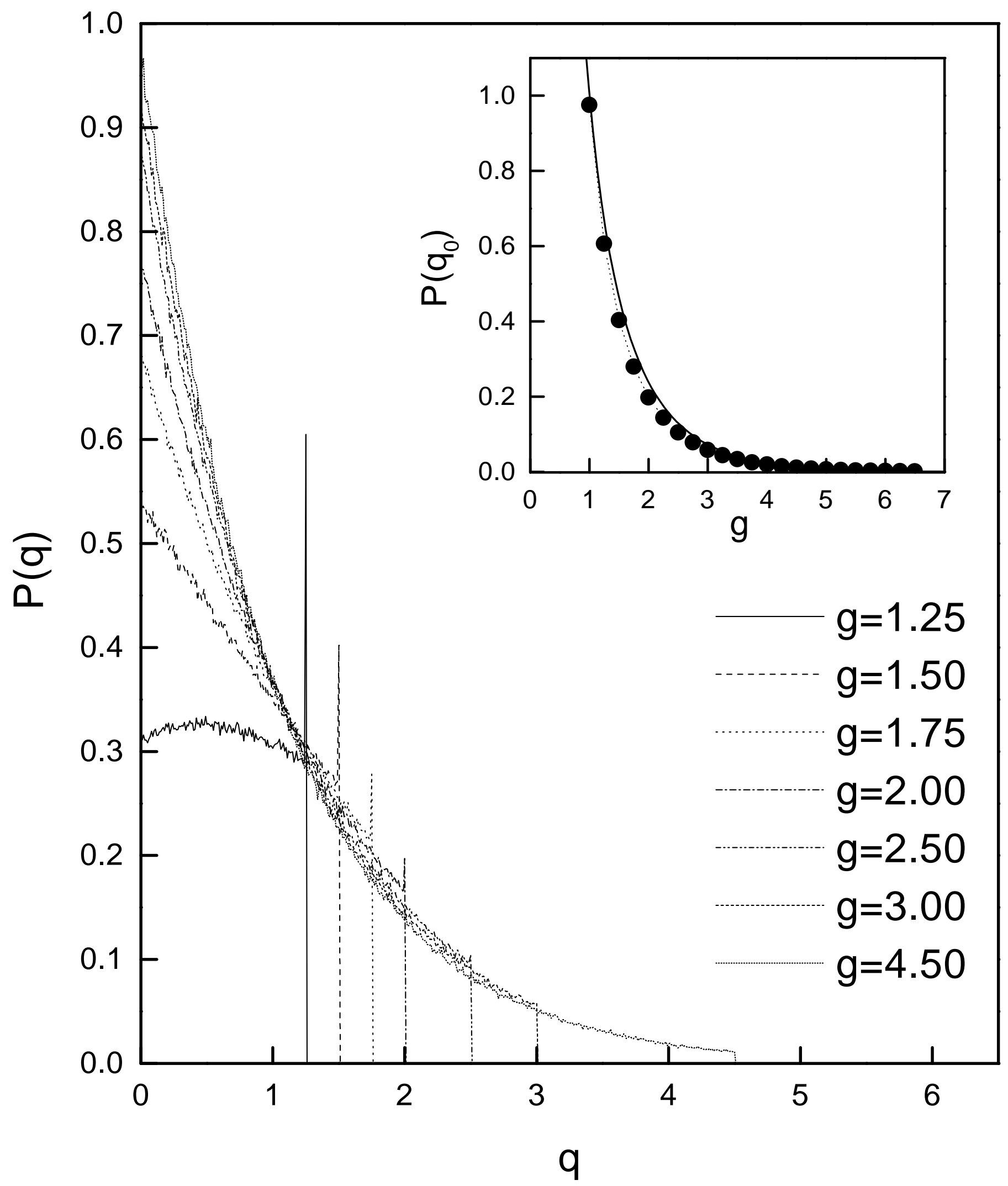

Fig. 2 


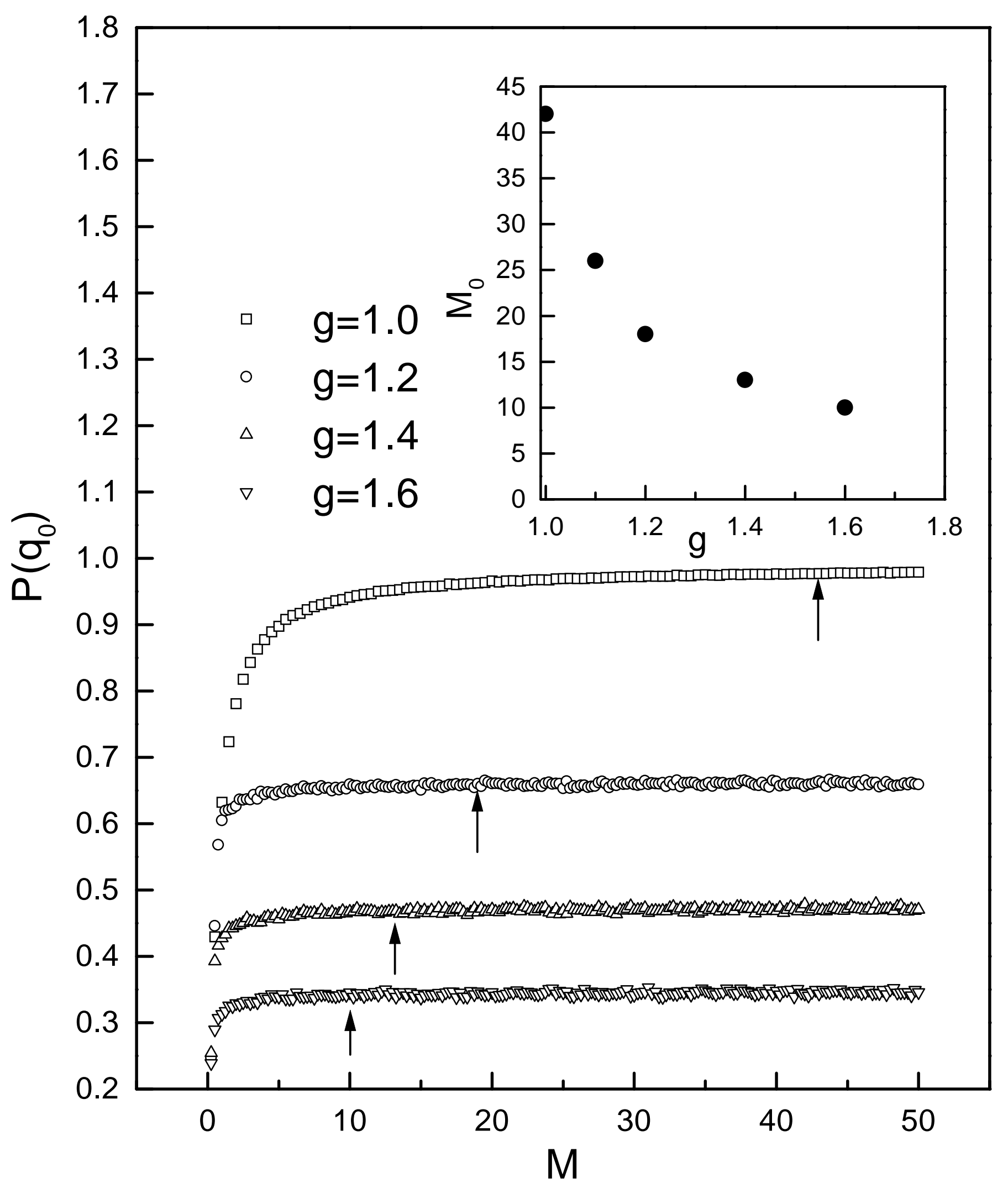

Fig. 3 